\title{
Immunogenicity Assessment of Lipegfilgrastim in Patients with Breast Cancer Receiving Chemotherapy
}

\author{
Linglong Zou, ${ }^{1}$ Anton Buchner, ${ }^{2}$ Martin Roberge, ${ }^{3}$ and Patrick M. Liu ${ }^{1}$ \\ ${ }^{1}$ Global Bioassays and Technology, Teva Pharmaceuticals, Inc., 145 Brandywine Parkway, West Chester, PA 19380, USA \\ ${ }^{2}$ Merckle GmbH, Graf-Arco-Straße 3, 89079 Ulm, Germany \\ ${ }^{3}$ CIRION BioPharma Research Inc., 3150 rue Delaunay, Laval, QC, Canada H7L 5E1
}

Correspondence should be addressed to Patrick M. Liu; patrick.liu@tevapharm.com

Received 23 March 2016; Accepted 23 May 2016

Academic Editor: Frank-Peter Theil

Copyright (C) 2016 Linglong Zou et al. This is an open access article distributed under the Creative Commons Attribution License, which permits unrestricted use, distribution, and reproduction in any medium, provided the original work is properly cited.

\begin{abstract}
Lipegfilgrastim is a long-acting, once-per-cycle, glycopegylated recombinant granulocyte colony-stimulating factor (G-CSF) used to prevent neutropenia in patients receiving myelosuppressive chemotherapy. This integrated analysis examined the immunogenicity of lipegfilgrastim and its potential clinical impact in two double-blind randomized studies (phases II and III) of patients with breast cancer receiving chemotherapy. Serum samples were analyzed using sequential assays for screening, confirmation, antibody titer, and characterization of antidrug antibodies (ADA). Neutropenia-related efficacy measures were reviewed for each ADA-positive patient. Among 255 patients receiving lipegfilgrastim (154 in phase II, 101 in phase III) and 155 patients receiving pegfilgrastim (54 in phase II, 101 in phase III), the incidence of treatment-emergent ADA was low and similar between the lipegfilgrastim (phase II: 1.3\%; phase III: 1.0\%) and pegfilgrastim (phase II: 1.9\%; phase III: $1.0 \%$ ) arms. None of the treatment-emergent ADA-positive samples exhibited neutralizing activity against lipegfilgrastim, pegfilgrastim, or glycosylated GCSF in a cell-based neutralizing antibody assay. No changes were observed in neutropenia-related efficacy measures among ADApositive patients, and no treatment-related hypersensitivity or anaphylaxis occurred. These results indicate that there is no apparent impact of ADA on lipegfilgrastim efficacy and safety.
\end{abstract}

\section{Introduction}

Granulocyte colony-stimulating factor (G-CSF) is an endogenous growth factor that promotes neutrophil production, maturation, survival, and activity [1]. Recombinant G-CSFs, such as filgrastim and pegfilgrastim, are used commonly for the prevention and treatment of neutropenia in patients receiving myelosuppressive chemotherapy [2-4].

Filgrastim requires daily administration to maintain therapeutic levels because of its relatively short half-life. Conjugating filgrastim to polyethylene glycol (PEG; pegylation yielding pegfilgrastim) reduces renal clearance and extends the drug's half-life such that it need be administered only once per chemotherapy treatment cycle, with efficacy and safety comparable to those of daily filgrastim [5-7].

Lipegfilgrastim (Lonquex; Teva Pharmaceuticals Ltd.) is a recombinant human G-CSF that is glycopegylated in a site-specific manner, resulting in greater structural homogeneity, with pharmacological properties slightly different from those of pegfilgrastim in healthy volunteers. Specifically, lipegfilgrastim provided a longer-lasting increase in absolute neutrophil count (ANC) compared with pegfilgrastim at an equivalent dose, without increasing the peak ANC values [8]. The noninferiority of lipegfilgrastim to pegfilgrastim in the treatment of severe neutropenia was demonstrated in a randomized, double-blind, active-controlled, phase III trial evaluating the efficacy and safety of lipegfilgrastim in 202 chemotherapy naive patients with breast cancer [9]. Lipegfilgrastim was approved in the European Union in 2013 as onceper-cycle, fixed-dose prophylaxis for severe neutropenia.

Immunogenicity is a potential concern for any biological product, and its assessment is one of the most critical elements for the development of such products. Antidrug antibody (ADA) production, as an unwanted immune response 
due to product immunogenicity, may lead to serious safety consequences that manifest as hypersensitivity responses such as anaphylaxis and development of cross-reactive neutralizing antibodies (NAbs) to endogenous proteins $[10,11]$. Recombinant G-CSFs, including filgrastim and pegfilgrastim, have been shown to elicit ADA in a minority of patients $[12,13]$.

The objective of this analysis was to assess the immunogenicity of lipegfilgrastim and its potential clinical impact using data from phase II dose-finding trial and phase III noninferiority trial conducted with patients with breast cancer receiving chemotherapy.

\section{Methods}

2.1. Study Design and Treatments. Immunogenicity assessments were performed on blood samples collected during two independent clinical studies $[9,14]$. The first study was a phase II, double-blind, randomized, dose-optimization study that evaluated the efficacy, safety, pharmacokinetics, and immunogenicity of drug treatments in 208 breast cancer patients undergoing myelosuppressive chemotherapy. Patients were assigned $1: 1: 1: 1$ to receive lipegfilgrastim (3.0, 4.5 , or $6.0 \mathrm{mg}$ administered via subcutaneous [SC] injection) or pegfilgrastim $(6.0 \mathrm{mg} \mathrm{SC})$ once per cycle while undergoing chemotherapy with intravenous doxorubicin $60 \mathrm{mg} / \mathrm{m}^{2}$ and docetaxel $75 \mathrm{mg} / \mathrm{m}^{2}$ [14]. The second study was a phase III, double-blind, randomized, noninferiority study in which 202 patients with breast cancer received either lipegfilgrastim (6.0 mg SC) or pegfilgrastim (6.0 mg SC) once per cycle while undergoing the same chemotherapy regimen [9].

In both studies, patients received intravenous doxorubicin/docetaxel administered on day 1 of four 21-day cycles. Lipegfilgrastim or pegfilgrastim was administered on day 2 of each cycle (i.e., 24 hours after chemotherapy was administered).

Blood samples were collected at several time points in each study: at baseline, prior to each chemotherapy cycle, at the end of treatment (day 85), and on posttreatment followup days 180 and 360 .

2.2. Study Populations. Eligible patients ( $\geq 18$ years of age) had a diagnosis of stage II, III, or IV breast cancer, were chemotherapy naive, had a baseline ANC of at least $1.5 \times$ $10^{9} / \mathrm{L}$ and a platelet count of $100 \times 10^{9} / \mathrm{L}$ or greater, and had an Eastern Cooperative Oncology Group performance status of 2 or less $[9,14]$. Exclusion criteria included known hypersensitivity to filgrastim or pegfilgrastim or exposure to those agents prior to randomization, prior malignancy within 5 years, radiation therapy within 4 weeks of randomization, or long-term use of oral corticosteroids.

The overall population comprised 208 patients from the phase II study (54 pegfilgrastim, 154 lipegfilgrastim) and 202 patients from the phase III study (101 in each treatment arm), for a total of 410 patients. Patient demographics and baseline clinical characteristics were matched between treatment groups within each study and have been reported elsewhere $[9,14]$. The mean ages were similar between

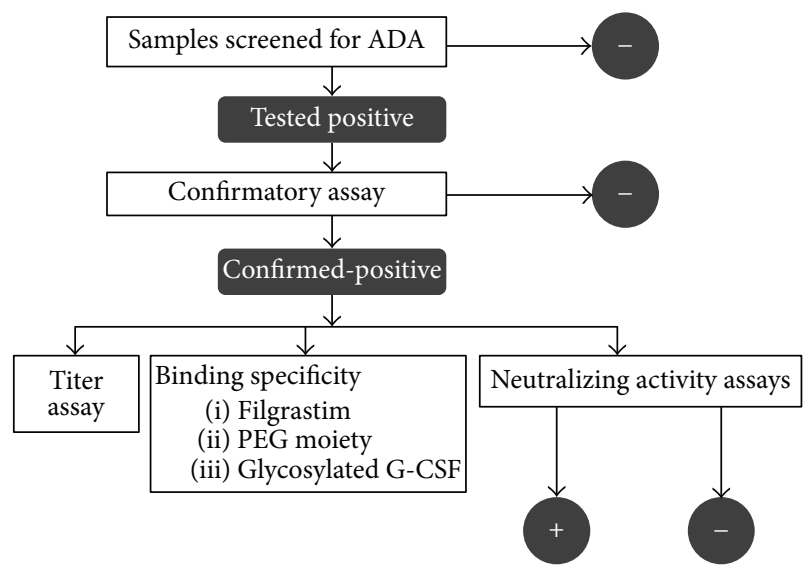

FIGURE 1: Sequential approach to assessing immunogenicity.

the lipegfilgrastim (51.4 years) and pegfilgrastim (50.5 years) groups. All patients were white, and all but three were female.

2.3. Immunogenicity Assays. A sequential cascade of validated assays was used to analyze ADA against lipegfilgrastim in patient serum samples (Figure 1). All samples were first tested in the screening assay. Samples screening positive were then analyzed using a confirmatory assay. After ADA confirmation, samples were characterized to determine binding specificity, antibody titers, and neutralizing activity.

2.3.1. Screening Assay. The screening assay used a ligandbinding principle in an electrochemiluminescent bridging format (Figure 2). Briefly, the bivalent property of ADA allows for simultaneous binding of a capture reagent (biotinlabeled lipegfilgrastim or pegfilgrastim) and a detection reagent (ruthenium-labeled lipegfilgrastim or pegfilgrastim). In the presence of a read buffer containing tripropylamine, an electrical current causes the captured ruthenium to emit measureable light. Signal was measured using a Sector Imager 6000 Analyzer (Meso Scale Discovery). The relative sensitivity of the screening assay was $10 \mathrm{ng} / \mathrm{mL}$ ADA to lipegfilgrastim and $45 \mathrm{ng} / \mathrm{mL}$ ADA to pegfilgrastim, determined using affinity-purified rabbit anti-lipegfilgrastim and rabbit anti-pegfilgrastim antibodies, respectively, as surrogate positive controls. Assay signals from a panel of samples from study drug-naive breast cancer patients were used for the determination of the assay cut-point factor, which in conjunction with the negative control was used to calculate the cut-point for each assay run. Cut-point factors with a false-positive rate set at $5 \%$ were determined using statistical methodology described elsewhere $[15,16]$. A sample with an assay signal at or above the cut-point was considered as having screened positive.

2.3.2. Confirmatory Assay. Samples that tested positive in the screening assay were subsequently analyzed in an immunocompetition assay for confirmation. Binding was measured with and without the study drug (lipegfilgrastim or pegfilgrastim) in solution phase, and the ratio of binding signal was 


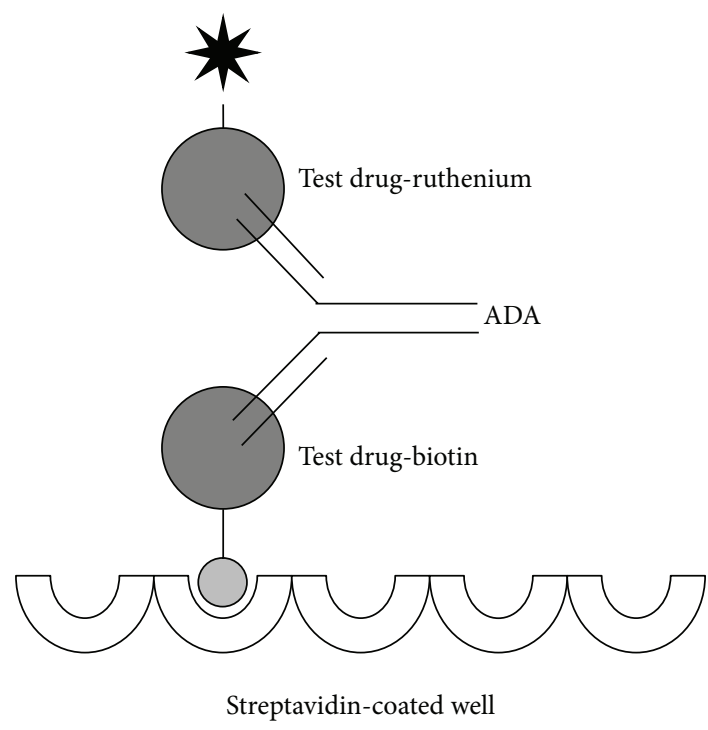

FIGURE 2: Schematic presentation of the electrochemiluminescent bridging immunoassay. Patient samples were diluted at the minimum required dilution, mixed with biotin- and rutheniumconjugated test drug (lipegfilgrastim or pegfilgrastim) and the complex formed by antidrug antibodies (ADA). The drug conjugates were captured on a streptavidin-coated assay plate. In the presence of a read buffer containing tripropylamine and upon application of an electrical potential, the ruthenium tag emits light.

calculated and expressed as a percentage of signal inhibition. A cut-point with a false-positive rate set at $1 \%$ was determined for each drug using commercially available serum samples from treatment-naive cancer patients in accordance with statistical methods $[15,16]$. Samples were identified as confirmed-positive if the percentage of signal inhibition was greater than or equal to the confirmatory cut-point for lipegfilgrastim (28.5\%) or pegfilgrastim (28.2\%).

\subsubsection{Characterization Assays. Confirmed-positive samples} were subsequently characterized to evaluate the ADA binding specificity. Binding signals were determined in the presence of unlabeled filgrastim, the PEG portion of lipegfilgrastim (cPEG), or glycosylated G-CSF (glycoG-CSF; Granocyte ${ }^{\varpi}$ Chugai Pharma), which is an analog of endogenous G-CSF. The cut-points (\% signal inhibition) for binding specificity were determined statistically (with false-positive rate set at $1 \%$ ), using commercially available serum samples from treatment-naive cancer patients in accordance with statistical methodology $[15,16]$, to be $22.0 \%$ for filgrastim, $19.4 \%$ for cPEG, and $17.5 \%$ for glycoG-CSF.

All confirmed-positive serum samples were also subjected to a semiquantitative titer assay. The titer was defined as the logarithm-transformed highest dilution factor resulting in a signal at the screening cut-point.

2.3.4. NAb Assays and Clinical Assessment. Neutralizing activity was assessed in the confirmed-positive ADA samples using a cell-based proliferation assay that tested the ability of the serum samples to inhibit various G-CSF (glycoG-CSF, lipegfilgrastim, or pegfilgrastim) stimulated proliferation of NSF-60 cells in vitro measured using WST-1 reagent. Samples were also tested in the absence of any G-CSF inducer to detect the presence of nonspecific cell growth that could result in false-negative neutralizing activity. Samples that inhibited any G-CSF inducers in the NAb assay underwent specificity testing in which proliferation was stimulated by murine interleukin-3, an inducer not specific to G-CSF activity; neutralization of this proliferation indicated nonspecific inhibition.

The cut-point for positivity was defined as an optical density (OD) ratio (OD sample: OD viability control) less than or equal to 0.824 for lipegfilgrastim inducer, 0.761 for pegfilgrastim inducer, and 0.821 for glycoG-CSF inducer. These cut-points were established statistically with the falsepositive rate set at 5\%. A cut-point with a multiplicative correction factor of 1.402 ( $1 \%$ false-positive rate) was established in accordance with statistical methods $[15,16]$ using commercially available serum samples from treatment-naive cancer patients when samples were tested in the absence of inducer.

Clinical measures were examined for all patients with confirmed-positive samples of ADA to lipegfilgrastim or pegfilgrastim for a possible correlation between the presence of ADA and the potential clinical impact of immunogenicity.

2.4. Efficacy Measurements. The primary efficacy measure was the duration of severe neutropenia (DSN), defined as the number of days with grade 4 neutropenia $(\mathrm{ANC}<0.5 \times$ $10^{9} / \mathrm{L}$ ) in each treatment cycle. A secondary efficacy measure was the incidence of febrile neutropenia, defined as axillary body temperature greater than $38.5^{\circ} \mathrm{C}$ for more than 1 hour and ANC less than $0.5 \times 10^{9} / \mathrm{L}$ across all cycles. Additional measures included ANC area under the concentration-time curve, maximum ANC, and mean depth of the ANC nadir.

\section{Results}

3.1. ADA Incidence. A total of 208 patients (54 pegfilgrastim and 154 lipegfilgrastim) were investigated in the phase II study and 202 patients in the phase III study. Results from the patients with confirmed ADA are summarized in Table 1. In the phase II study, 2 of the 154 lipegfilgrastim-treated patients (Table 1, patients 1 and 2) had treatment-emergent ADA, representing an incidence of $1.3 \%$. These patients had confirmedpositive samples at a single postdose time point (day 85 or day 360), indicating that the response was transient. There were seven patients with predose ADA, including three with positive samples at both baseline and postdose time points (Table 1, patients 3-5) and four with positive samples at baseline only (Table 1, patients 6-9). One of 54 pegfilgrastimtreated patients (Table 1 , patient 10) had treatment-emergent $\mathrm{ADA}$, representing an incidence of $1.9 \%$. In this patient, the ADA-positive sample occurred only at day 85 . There were two patients in the pegfilgrastim group with positive ADA samples observed at baseline only (Table 1, patients 11 and 12).

In the phase III study, 1 of the 101 lipegfilgrastim-treated patients (Table 1, patient 13) had treatment-emergent ADA, with positive samples at days 180 and 360. This reflects 
TABLE 1: Summary of patients with ADA-positive samples.

\begin{tabular}{|c|c|c|c|c|c|c|c|c|c|}
\hline \multicolumn{10}{|c|}{ Phase II study } \\
\hline \multirow{2}{*}{ Patient } & \multirow{2}{*}{ Treatment group } & \multicolumn{8}{|c|}{ Visit } \\
\hline & & $\mathrm{BL}$ & C2D1 & C3D1 & C4D1 & D85 & $\mathrm{D} 180$ & D360 & ET \\
\hline 1 & Lipegfilgrastim & $\mathrm{Neg}$ & $\mathrm{Neg}$ & $\mathrm{Neg}$ & $\mathrm{Neg}$ & Pos & $N A$ & $N A$ & $N A$ \\
\hline 2 & Lipegfilgrastim & $N A$ & $N A$ & $N A$ & $N A$ & $N A$ & $N A$ & Pos & $N A$ \\
\hline 3 & Lipegfilgrastim & Pos & Pos & Pos & Pos & Pos & Pos & Pos & NA \\
\hline 4 & Lipegfilgrastim & Pos & Pos & Pos & Pos & Pos & Pos & Neg & NA \\
\hline 5 & Lipegfilgrastim & Pos & Pos & NA & NA & NA & Pos & Pos & Neg \\
\hline 6 & Lipegfilgrastim & Pos & NA & NA & NA & NA & NA & NA & NA \\
\hline 7 & Lipegfilgrastim & Pos & NA & NA & NA & NA & NA & NA & NA \\
\hline 8 & Lipegfilgrastim & Pos & NA & NA & NA & NA & NA & Neg & NA \\
\hline 9 & Lipegfilgrastim & Pos & NA & NA & NA & NA & NA & NA & NA \\
\hline 10 & Pegfilgrastim & $N A$ & $N A$ & $N A$ & $N A$ & Pos & $N A$ & $N A$ & $N A$ \\
\hline 11 & Pegfilgrastim & Pos & NA & NA & NA & NA & NA & NA & NA \\
\hline 12 & Pegfilgrastim & Pos & NA & NA & NA & NA & NA & NA & NA \\
\hline \multicolumn{10}{|c|}{ Phase III study } \\
\hline \multirow{2}{*}{ Patient } & \multirow{2}{*}{ Treatment group } & \multicolumn{8}{|c|}{ Visit } \\
\hline & & S & $\mathrm{BL}$ & $\mathrm{C} 2 \mathrm{D} 1$ & C3D1 & $\mathrm{C} 4 \mathrm{D} 1$ & D85 & $\mathrm{D} 180$ & D360 \\
\hline 13 & Lipegfilgrastim & $N A$ & $N A$ & $N A$ & $N A$ & $N A$ & $N A$ & Pos & Pos \\
\hline 14 & Lipegfilgrastim & NA & Pos & Pos & Pos & Pos & Neg & Neg & $\mathrm{Neg}$ \\
\hline 15 & Lipegfilgrastim & NA & Pos & Pos & Neg & Neg & Neg & Neg & $\mathrm{Neg}$ \\
\hline 16 & Lipegfilgrastim & NA & Pos & NA & NA & NA & NA & NA & NA \\
\hline 17 & Pegfilgrastim & $N A$ & $N A$ & $N A$ & $N A$ & $N A$ & $N A$ & $N A$ & Pos \\
\hline 18 & Pegfilgrastim & NA & Pos & NA & NA & NA & NA & NA & NA \\
\hline 19 & Pegfilgrastim & Pos & NA & NA & NA & NA & NA & NA & NA \\
\hline 20 & Pegfilgrastim & NA & Pos & NA & NA & NA & NA & NA & NA \\
\hline 21 & Pegfilgrastim & NA & Pos & NA & NA & NA & NA & NA & NA \\
\hline 22 & Pegfilgrastim & NA & Pos & Neg & NA & NA & NA & NA & NA \\
\hline 23 & Pegfilgrastim & NA & Pos & NA & NA & NA & NA & NA & NA \\
\hline
\end{tabular}

Note: table includes only patients with ADA-positive samples.

ADA: antidrug antibody; BL: baseline; C2D1: cycle 2 day 1; C3D1: cycle 3 day 1; C4D1: cycle 4 day 1; D85: day 85; D180: day 180; D360: day 360; NA: screened negative sample; Neg: confirmed-negative sample; Pos: confirmed-positive sample; S: screening (predose) time point.

Italic font indicates patient with treatment-emergent ADA.

an ADA incidence of $1.0 \%$. There were three patients with predose ADA, including one who had a positive sample at baseline only (Table 1 , patient 16) and two who had positive samples at both baseline and at least a postdose time point (Table 1, patients 14 and 15). Among 101 pegfilgrastim-treated patients in this study, one had treatment-emergent ADA, with a positive postdose sample at day 360 only (Table 1 , patient 17). This also represents an ADA incidence of $1.0 \%$. Six patients (Table 1, patients 18-23) had predose ADA, with positive samples at baseline only.

3.2. ADA Characterization and Titer. The binding specificity and titer of ADA were determined in samples from lipegfilgrastim-treated patients with predose or treatmentemergent ADA. Twenty-two confirmed-positive samples from nine lipegfilgrastim-treated patients in the phase II trial were tested using filgrastim, cPEG, or glycoG-CSF competitors to identify ADA binding specificity for the G-CSF moiety of lipegfilgrastim, the PEG moiety of lipegfilgrastim, and endogenous G-CSF, respectively (Table 2).
As noted above, only two patients in the lipegfilgrastimtreated group exhibited treatment-emergent ADA. One had a positive sample on day 85 , with an antibody titer of 0.6 against cPEG only (Table 2). The other patient had the positive sample on day 360 . The antibody titer was undetectable in this sample and showed no recognition of filgrastim, glycoGCSF, or CPEG. Five of the seven remaining patients showed predose ADA; two of these five had positive postdose ADA samples as well. One of these two patients had antibodies recognizing filgrastim and glycoG-CSF without detectable titer throughout the duration of the study. The other patient had antibodies recognizing filgrastim and CPEG whose titer diminished over time from 0.9 to 0.3 . The remaining three of these five patients had predose antibodies recognizing filgrastim and CPEG but no detectable postdose antibody titer.

From the phase III study, nine confirmed-positive samples from four patients underwent the same characterization and titer assays. One lipegfilgrastim-treated patient with a confirmed-positive sample had possible treatment-emergent 
TABLE 2: ADA titer and binding specificity of ADA-positive samples from lipegfilgrastim-treated patients.

\begin{tabular}{|c|c|c|c|c|c|c|c|c|}
\hline \multirow{2}{*}{ Patient } & \multirow{2}{*}{ Competitor/titer } & \multicolumn{7}{|c|}{ Time point } \\
\hline & & $\mathrm{BL}$ & C2D1 & C3D1 & C4D1 & D85 & D180 & D360 \\
\hline \multicolumn{9}{|c|}{ Phase II study } \\
\hline \multirow{4}{*}{1} & Filgrastim & $N A$ & $N A$ & $N A$ & $N A$ & $\mathrm{Neg}$ & $N A$ & $N A$ \\
\hline & glycoG-CSF & $N A$ & $N A$ & $N A$ & $N A$ & $\mathrm{Neg}$ & $N A$ & $N A$ \\
\hline & $c P E G$ & $N A$ & $N A$ & $N A$ & $N A$ & Pos & $N A$ & $N A$ \\
\hline & Titer & NA & $N A$ & $N A$ & $N A$ & 0.6 & $N A$ & NA \\
\hline \multirow{4}{*}{2} & Filgrastim & $N A$ & $N A$ & $N A$ & $N A$ & $N A$ & $N A$ & $\mathrm{Neg}$ \\
\hline & glycoG-CSF & $N A$ & $N A$ & $N A$ & $N A$ & $N A$ & $N A$ & $\mathrm{Neg}$ \\
\hline & $c P E G$ & $N A$ & $N A$ & $N A$ & $N A$ & $N A$ & $N A$ & $\mathrm{Neg}$ \\
\hline & Titer & $N A$ & $N A$ & $N A$ & $N A$ & $N A$ & $N A$ & $0^{\circ}$ \\
\hline \multirow{4}{*}{3} & Filgrastim & Pos & Pos & Pos & Pos & Pos & Pos & Pos \\
\hline & glycoG-CSF & Pos & Pos & Pos & Pos & Pos & Pos & Pos \\
\hline & cPEG & Neg & Neg & Neg & Neg & $\mathrm{Neg}$ & Neg & Neg \\
\hline & Titer & 0 & 0 & 0 & 0 & 0 & 0 & 0 \\
\hline \multirow{4}{*}{4} & Filgrastim & Pos & Pos & Pos & Pos & Neg & Pos & NA \\
\hline & glycoG-CSF & Neg & Neg & Neg & Pos & Pos & Pos & NA \\
\hline & cPEG & Pos & Pos & Pos & Pos & Pos & Pos & NA \\
\hline & Titer & 0.3 & 0.9 & 0.6 & 0.6 & 0.6 & 0.3 & NA \\
\hline \multirow{4}{*}{5} & Filgrastim & NSQ & Neg & NA & NA & NA & Neg & Neg \\
\hline & glycoG-CSF & NSQ & $\mathrm{Neg}$ & NA & NA & NA & $\mathrm{Neg}$ & $\mathrm{Neg}$ \\
\hline & cPEG & NSQ & Pos & NA & NA & NA & Pos & Pos \\
\hline & Titer & NSQ & 0 & NA & NA & NA & 0 & 0 \\
\hline \multirow{4}{*}{6} & Filgrastim & Neg & NA & NA & NA & NA & NA & NA \\
\hline & glycoG-CSF & $\mathrm{Neg}$ & NA & NA & NA & NA & NA & NA \\
\hline & cPEG & Neg & NA & NA & NA & NA & NA & NA \\
\hline & Titer & $0.6^{\circ}$ & NA & NA & NA & NA & NA & NA \\
\hline \multirow{4}{*}{7} & Filgrastim & Pos & NA & NA & NA & NA & NA & NA \\
\hline & glycoG-CSF & Neg & NA & NA & NA & NA & NA & NA \\
\hline & cPEG & Pos & NA & NA & NA & NA & NA & NA \\
\hline & Titer & 1.5 & NA & NA & NA & NA & NA & NA \\
\hline \multirow{4}{*}{8} & Filgrastim & Pos & NA & NA & NA & NA & NA & NA \\
\hline & glycoG-CSF & Neg & NA & NA & NA & NA & NA & NA \\
\hline & cPEG & Pos & NA & NA & NA & NA & NA & NA \\
\hline & Titer & 0.3 & NA & NA & NA & NA & NA & NA \\
\hline \multirow{4}{*}{9} & Filgrastim & Pos & NA & NA & NA & NA & NA & NA \\
\hline & glycoG-CSF & Neg & NA & NA & NA & NA & NA & NA \\
\hline & cPEG & Pos & NA & NA & NA & NA & NA & NA \\
\hline & Titer & 0 & NA & NA & NA & NA & NA & NA \\
\hline \multicolumn{9}{|c|}{ Phase III study } \\
\hline \multirow{4}{*}{13} & Filgrastim & $N A$ & $N A$ & $N A$ & $N A$ & $N A$ & Pos & Pos \\
\hline & glycoG-CSF & $N A$ & $N A$ & $N A$ & $N A$ & $N A$ & Pos & Pos \\
\hline & $c P E G$ & $N A$ & $N A$ & $N A$ & $N A$ & $N A$ & $\mathrm{Neg}$ & $\mathrm{Neg}$ \\
\hline & Titer & $N A$ & $N A$ & $N A$ & $N A$ & $N A$ & 1.2 & 2.1 \\
\hline \multirow{4}{*}{14} & Filgrastim & Neg & $\mathrm{Neg}$ & $\mathrm{Neg}$ & $\mathrm{Neg}$ & NA & NA & NA \\
\hline & glycoG-CSF & Neg & Neg & $\mathrm{Neg}$ & $\mathrm{Neg}$ & NA & NA & NA \\
\hline & CPEG & Pos & Pos & Pos & Pos & NA & NA & NA \\
\hline & Titer & 0.6 & 1.2 & 0.9 & 0.6 & NA & NA & NA \\
\hline \multirow{4}{*}{15} & Filgrastim & Pos & Neg & NA & NA & NA & NA & NA \\
\hline & glycoG-CSF & Pos & Pos & NA & NA & NA & NA & NA \\
\hline & cPEG & Neg & Neg & NA & NA & NA & NA & NA \\
\hline & Titer & 2.1 & $1.8^{\circ}$ & NA & NA & NA & NA & NA \\
\hline & Filgrastim & Pos & NA & NA & NA & NA & NA & NA \\
\hline & glycoG-CSF & Neg & NA & NA & NA & NA & NA & NA \\
\hline 16 & cPEG & Pos & NA & NA & NA & NA & NA & NA \\
\hline & Titer & 0.9 & NA & NA & NA & NA & NA & NA \\
\hline
\end{tabular}

ADA: antidrug antibody; BL: baseline; C2D1: cycle 2 day 1; C3D1: cycle 3 day 1; C4D1: cycle 4 day 1; cPEG: PEG portion of lipegfilgrastim; D85: day 85; D180: day 180; D360: day 360; glycoG-CSF: glycosylated granulocyte colony-stimulating factor; NA: screened negative sample or confirmed-negative one (not analyzed in characterization assay); Neg: confirmed-negative sample; NSQ: insufficient sample quantity for analysis; Pos: confirmed-positive sample. Italic font indicates patient with treatment-emergent ADA. 
TABLE 3: Evaluation of the potential impact of ADA on efficacy among patients with treatment-emergent ADA.

\begin{tabular}{|c|c|c|c|c|c|c|c|}
\hline Treatment & Patient & ADA + time point & & DSN (days) & ANC AUC & ANC maximum & ANC nadir \\
\hline \multicolumn{8}{|c|}{ Phase II study } \\
\hline \multirow{4}{*}{ Pegfilgrastim } & \multirow{4}{*}{10} & \multirow{4}{*}{ D85 } & Cycle 1 & 0 & 160.25 & 9.60 & 6.8 \\
\hline & & & Cycle 2 & 0 & 212.95 & 14.90 & 7.9 \\
\hline & & & Cycle 3 & 0 & 116.25 & 8.50 & 3.6 \\
\hline & & & Cycle 4 & 0 & 91.85 & 7.50 & 2.4 \\
\hline \multirow{8}{*}{ Lipegfilgrastim } & \multirow{4}{*}{2} & \multirow{4}{*}{ D360 } & Cycle 1 & 1 & 154.24 & 20.39 & 0.18 \\
\hline & & & Cycle 2 & 1 & 170.20 & 21.40 & 0.0 \\
\hline & & & Cycle 3 & 0 & 225.40 & 27.64 & 1.25 \\
\hline & & & Cycle 4 & 0 & 202.26 & 28.42 & 1.72 \\
\hline & \multirow{4}{*}{1} & \multirow{4}{*}{ D85 } & Cycle 1 & 0 & 127.80 & 17.00 & 0.8 \\
\hline & & & Cycle 2 & 0 & 147.25 & 9.60 & 2.2 \\
\hline & & & Cycle 3 & 0 & 121.60 & 8.30 & 0.7 \\
\hline & & & Cycle 4 & 0 & 179.55 & 16.70 & 1.3 \\
\hline \multicolumn{8}{|c|}{ Phase III study } \\
\hline \multirow{4}{*}{ Pegfilgrastim } & \multirow{4}{*}{17} & \multirow{4}{*}{ D85 } & Cycle 1 & 0 & 219.88 & 47.99 & 0.89 \\
\hline & & & Cycle 2 & 0 & 198.15 & 13.73 & 2.2 \\
\hline & & & Cycle 3 & 0 & 302.98 & 68.32 & 2.16 \\
\hline & & & Cycle 4 & 0 & 315.63 & 57.42 & 4.06 \\
\hline \multirow{4}{*}{ Lipegfilgrastim } & \multirow{4}{*}{13} & \multirow{4}{*}{ D360 } & Cycle 1 & 2 & 160.16 & 26.14 & 0.25 \\
\hline & & & Cycle 2 & 0 & 257.95 & 51.92 & 1.25 \\
\hline & & & Cycle 3 & 0 & 300.24 & 58.91 & 1.2 \\
\hline & & & Cycle 4 & 0 & 291.36 & 68.75 & 1.45 \\
\hline
\end{tabular}

ADA: antidrug antibody; ANC: absolute neutrophil count; AUC: area under the curve; D85: day 85; D360: day 360; DSN: duration of severe neutropenia.

antibody induction, recognizing filgrastim and glycoG-CSF, but not CPEG, on both day 180 and day 360 (Table 2). The sample also showed antibody titers of 1.2 and 2.1 for days 180 and 360, respectively. The remaining three patients had confirmed-positive samples at baseline (i.e., predose ADA). One of these patients had antibodies recognizing CPEG at baseline, with a titer of 0.6 that increased to 1.2 in cycle 2 but diminished to undetectable levels by day 85 . Another patient had antibodies recognizing filgrastim and glycoGCSF at baseline, with a titer of 2.1; the antibodies for glycoGCSF persisted to cycle 2, with a titer of 1.8. The third patient had antibodies against filgrastim and CPEG, with a titer of 0.9 and no detectable postdose antibodies.

3.3. Neutralizing Activity of ADA. Among patients identified as having treatment-emergent $\mathrm{ADA}$, no postdose sample from either treatment group in each study tested positive for NAb activity against lipegfilgrastim, pegfilgrastim, or glycoGCSF in the cell-based neutralizing antibody assay.

3.4. Clinical Impact Assessments. Clinical efficacy measures for patients with confirmed-positive, treatment-emergent ADA are summarized in Table 3. Neither ANC nor DSN values changed in these patients after initiation of chemotherapy, and no patient experienced febrile neutropenia. No drugrelated events with the Medical Dictionary for Regulatory Activities preferred term "drug hypersensitivity" or "hypersensitivity" and no anaphylactic reactions were reported.

The effect of ADA on the pharmacokinetics of lipegfilgrastim was investigated in a pooled analysis of data from patients with breast cancer and patients with non-small cell lung cancer in the phase III study. Only two patients for whom pharmacokinetic data are available tested positive for ADA, and no decrease in exposure, as indicated by the predicted area under the curve data, to lipegfilgrastim was observed in these patients (data not shown). A pharmacodynamics analysis with a CD34+ endpoint conducted with adult patients for all lipegfilgrastim doses found only two patients with positive ADA response. The CD34+ values for these two patients were similar to those from ADA-negative subjects (data not shown).

\section{Discussion}

The objective of this analysis was to assess the immunogenicity of lipegfilgrastim in patients receiving chemotherapy for breast cancer. In both phase II and phase III studies, the incidence of treatment-emergent ADA was low and was similar between the lipegfilgrastim (phase II: $2 / 154=1.3 \%$; phase III: $1 / 101=1.0 \%$ ) and pegfilgrastim (phase II: $1 / 54=$ $1.9 \%$; phase III: $1 / 101=1.0 \%$ ) groups.

Among lipegfilgrastim-treated patients with treatmentemergent ADA, none of the postdose positive samples from either study exhibited NAb activity. Furthermore, there was no apparent impact of treatment-emergent ADA on key measures of clinical efficacy in these patients, including the duration of severe neutropenia or incidence of febrile neutropenia. The limited pharmacokinetic/pharmacodynamic data available for patients positive for ADA suggested no correlation between lipegfilgrastim exposure and ADA positivity. 
The results in this study are consistent with literature reporting the immunogenicity of pegfilgrastim, in which a small number $(4 / 521 ; 0.77 \%)$ of pegfilgrastim-treated patients developed treatment-emergent ADA [13]. Similar to the current study, none of these ADA-positive patients showed evidence of NAb activity.

In the current analysis of ADA data from two clinical studies in patients with breast cancer, 10 of 255 (3.9\%) lipegfilgrastim-treated patients had positive samples at baseline, a percentage similar to that among pegfilgrastim-treated patients $(7 / 155 ; 4.5 \%)$. Moreover, these patients did not experience an increase in their preexisting antibody response, maintaining relatively low titer levels throughout the course of treatment. Previous studies, again possibly using different assay methodologies, detected anti-pegfilgrastim antibodies in baseline samples from nearly $6 \%$ of pegfilgrastim-treated patients with metastatic breast cancer [13]. Therefore, it is not unexpected that some preexisting anti-lipegfilgrastim antibodies were observed at baseline in the current analysis.

This analysis found a low incidence of treatment-emergent ADA in both lipegfilgrastim- and pegfilgrastim-treated patients with breast cancer and treated with doxorubicin and docetaxel. The presence of treatment-emergent ADA did not appear to impact the clinical efficacy of either treatment; this was expected, because none of the detected antibodies in patients with possible treatment-emergent antibodies were neutralizing. Similarly, 11 of 333 patients (3\%) developed ADA following filgrastim treatment in clinical studies, and no neutralizing response was observed in the 11 patients [12].

\section{Competing Interests}

Linglong Zou, Anton Buchner, and Patrick M. Liu are employees of Teva Pharmaceuticals and declare financial interests regarding the publication of this paper; Martin Roberge reports employment from CIRION BioPharma Research Inc., a contract research laboratory providing services to Teva Pharmaceuticals.

\section{Acknowledgments}

The study was supported by Teva Branded Pharmaceutical Products R\&D. Financial support for medical writing assistance from Pamela Foreman, Ph.D., and Ada Ao-Baslock, Ph.D., of Powered 4 Significance LLC was provided by Teva Branded Pharmaceutical Products R\&D.

\section{References}

[1] A. W. Roberts, "G-CSF: a key regulator of neutrophil production, but that's not all!," Growth Factors, vol. 23, no. 1, pp. 33-41, 2005.

[2] K. L. Cooper, J. Madan, S. Whyte, M. D. Stevenson, and R. L. Akehurst, "Granulocyte colony-stimulating factors for febrile neutropenia prophylaxis following chemotherapy: systematic review and meta-analysis," BMC Cancer, vol. 11, article 404, 2011.

[3] J. Crawford, C. Caserta, and F. Roila, "Hematopoietic growth factors: ESMO Clinical Practice Guidelines for the applications," Annals of Oncology, vol. 21, supplement 5, pp. v248-v251, 2010.
[4] D. C. Dale, "Colony-stimulating factors for the management of neutropenia in cancer patients," Drugs, vol. 62, supplement 1, pp. 1-15, 2002.

[5] T. Arvedson, J. O’Kelly, and B.-B. Yang, "Design rationale and development approach for pegfilgrastim as a long-acting granulocyte colony-stimulating factor," BioDrugs, vol. 29, no. 3, pp. 185-198, 2015.

[6] M. D. Green, H. Koelbl, J. Baselga et al., "A randomized double-blind multicenter phase III study of fixed-dose singleadministration pegfilgrastim versus daily filgrastim in patients receiving myelosuppressive chemotherapy," Annals of Oncology, vol. 14, no. 1, pp. 29-35, 2003.

[7] F. A. Holmes, S. E. Jones, J. O’Shaughnessy et al., "Comparable efficacy and safety profiles of once-per-cycle pegfilgrastim and daily injection filgrastim in chemotherapy-induced neutropenia: a multicenter dose-finding study in women with breast cancer," Annals of Oncology, vol. 13, no. 6, pp. 903-909, 2002.

[8] A. Buchner, A. Lammerich, A. Abdolzade-Bavil, U. Müller, and P. Bias, "Lipegfilgrastim: pharmacodynamics and pharmacokinetics for body-weight-adjusted and $6 \mathrm{mg}$ fixed doses in two randomized studies in healthy volunteers," Current Medical Research and Opinion, vol. 30, no. 12, pp. 2523-2533, 2014.

[9] I. Bondarenko, O. A. Gladkov, R. Elsaesser, A. Buchner, and P. Bias, "Efficacy and safety of lipegfilgrastim versus pegfilgrastim: a randomized, multicenter, active-control phase 3 trial in patients with breast cancer receiving doxorubicin/docetaxel chemotherapy," BMC Cancer, vol. 13, article 386, 2013.

[10] H. Frost, "Antibody-mediated side effects of recombinant proteins," Toxicology, vol. 209, no. 2, pp. 155-160, 2005.

[11] A. S. Rosenberg, "Immunogenicity of biological therapeutics: a hierarchy of concerns," Developments in Biologicals, vol. 112, pp. 15-21, 2003.

[12] Neupogen [Prescribing Information], Amgen, Thousand Oaks, Calif, USA, 2015.

[13] Neulasta [Prescribing Information], Amgen, Thousand Oaks, Calif, USA, 2016.

[14] A. Buchner, R. Elsässer, and P. Bias, "A randomized, doubleblind, active control, multicenter, dose-finding study of lipegfilgrastim (XM22) in breast cancer patients receiving myelosuppressive therapy," Breast Cancer Research and Treatment, vol. 148, no. 1, pp. 107-116, 2014.

[15] G. Shankar, V. Devanarayan, L. Amaravadi et al., "Recommendations for the validation of immunoassays used for detection of host antibodies against biotechnology products," Journal of Pharmaceutical and Biomedical Analysis, vol. 48, no. 5, pp. 12671281, 2008.

[16] H. W. Smith, M. Moxness, and R. Marsden, "Summary of confirmation cut point discussions," AAPS Journal, vol. 13, no. 2, pp. 227-229, 2011. 


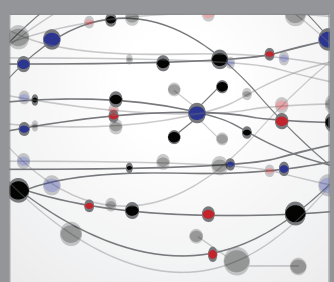

The Scientific World Journal
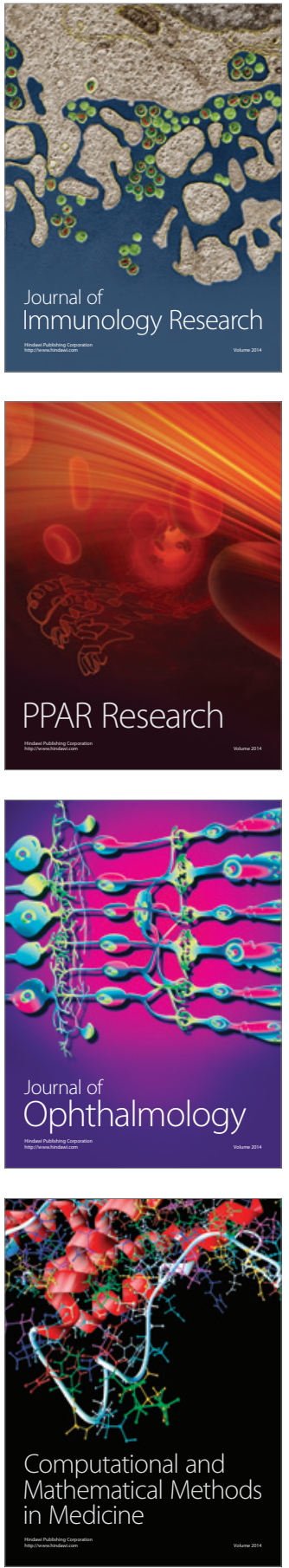

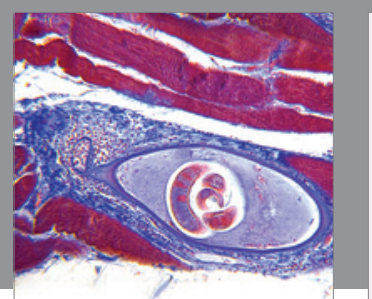

Gastroenterology Research and Practice

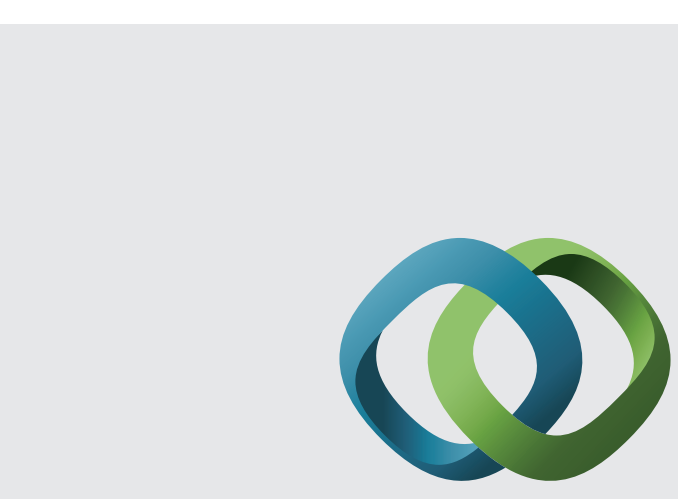

\section{Hindawi}

Submit your manuscripts at

http://www.hindawi.com
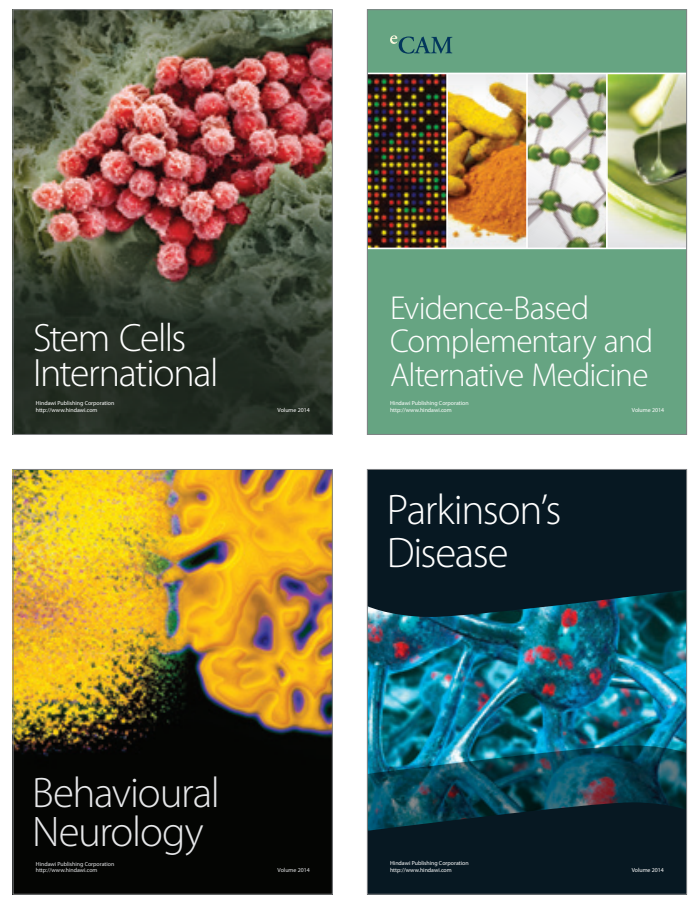
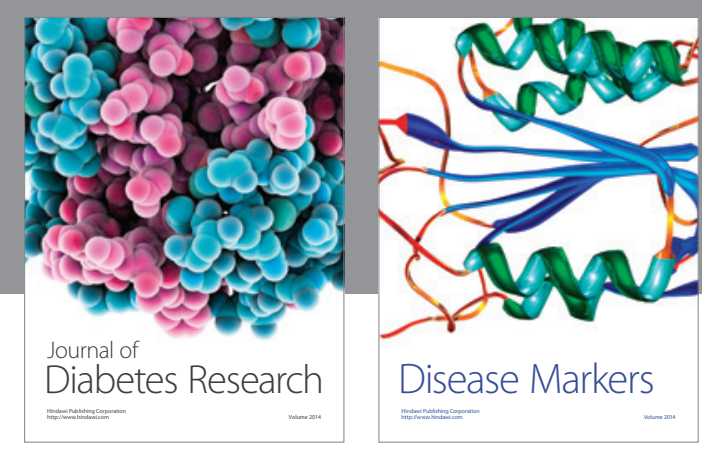

Disease Markers
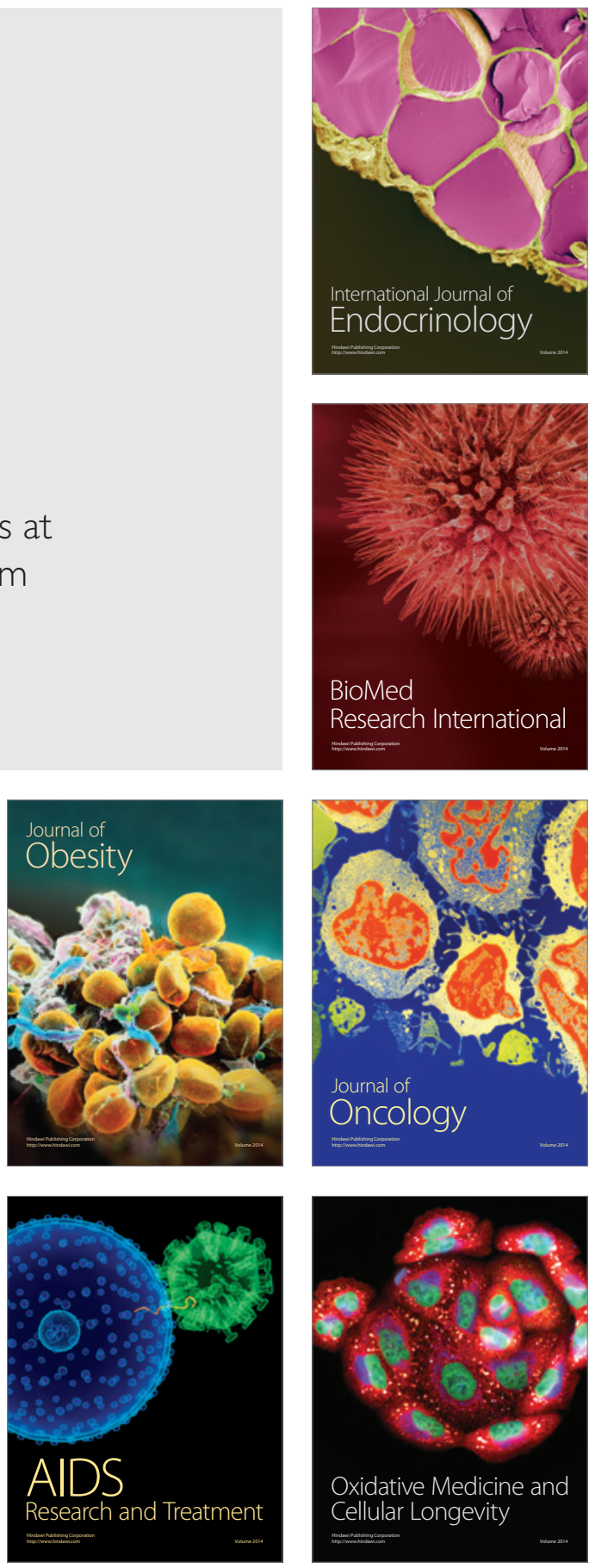